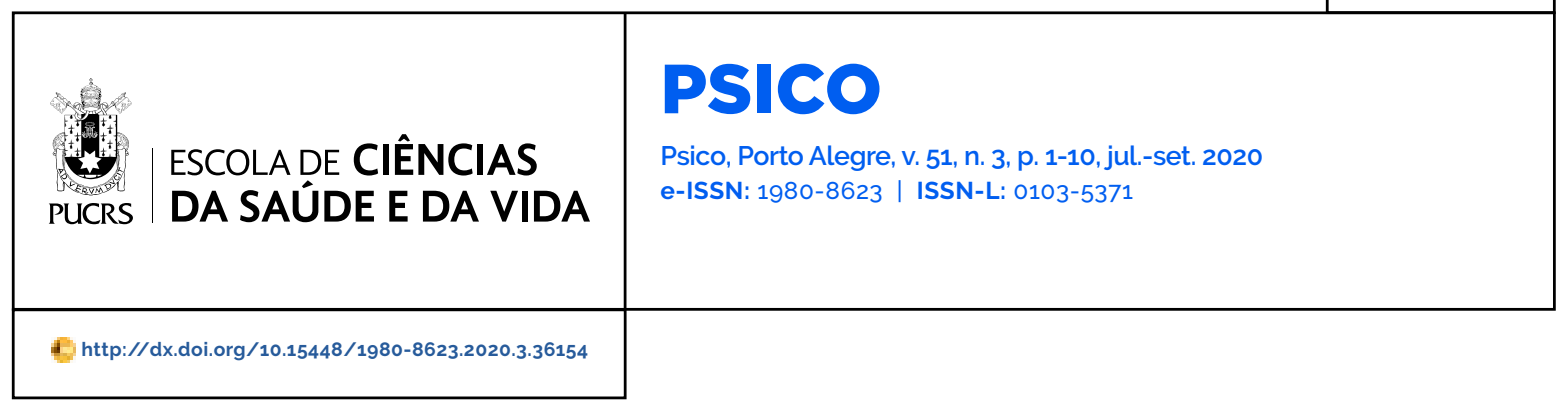

ARTIGOS

\title{
Estilos de aprendizagem e desempenho acadêmico de universitários de administração
}

\author{
Learning styles and academic performance of university students of business administration \\ Estilos de aprendizaje y rendimiento académico de universitarios de administración
}

Rodrigo Hipólito Roza
orcid.org/0000-0002-7162-4908

rodrigo.roza@gmail.com

\section{Acácia Aparecida Angeli dos Santos ${ }^{2}$ orcid.org/0000-0002-8599-7465 acacia.angeli@gmail.com}

Recebido em: 28/10/2019 Aprovado em: 25/2/2020 Publicado em: 21/01/2021.
Resumo: Este estudo teve o objetivo de investigar a relação entre os estilos de aprendizagem em situações de uso das Tecnologias da Informação e Comunicação (TICs) e o desempenho acadêmico de estudantes universitários de administração. A amostra foi composta por 286 estudantes de administração, com idades entre 18 e 43 anos ( $M=21,97$; $D P=3,10)$, sendo 53,15\% do gênero feminino. Os dados sobre estilos foram coletados por meio da Escala de Estilos de Aprendizagem em Situações de Uso de Tecnologias; os dados sobre desempenho acadêmico, por sua vez, foram obtidos com base em avaliações realizadas em uma disciplina de graduação em administração, utilizando a metodologia ativa de aprendizagem baseada em problemas (PBL). Os resultados indicaram que o desempenho acadêmico dos estudantes de administração não dependeu de seus estilos de aprendizagem, possivelmente em função da diversidade de atividades realizadas no âmbito da metodologia ativa adotada.

Palavras-chave: estilo de aprendizagem, desempenho acadêmico, tecnologia da informação, universitários, administração de empresas

Abstract: This study aimed to investigate the relationship between learning styles in situations of use of Information and Communication Technologies (ICTs) and the academic performance of university students of business administration. The sample consisted of 286 students of administration, aged between 18 and 43 years $(M=21.97 ; S D=3.10)$, being $53.15 \%$ female. The data on styles were collected through the Scale of Learning Styles in Technology Use Situations; the data on academic performance were obtained based on assessments of an undergraduate course in management using the active problem-based learning methodology (PBL). The results indicated that the academic performance of management students did not depend on their learning styles, possibly due to the diversity of activities carried out within the scope of the active methodology adopted.

Keywords: learning styles, academic performance, information technology, university student, business administration

Resumen: Este estudio tuvo el objetivo de investigar la relación entre los estilos de aprendizaje en situaciones de uso de las Tecnologias de la Información y Comunicación (TICs) y el desempeño académico de estudiantes universitarios de administración. La muestra fue compuesta por 286 estudiantes de administración, con edades entre 18 y 43 años $(M=21,97, D P=3,10)$, siendo el 53,15\% del género femenino. Los datos sobre estilos fueron recolectados por medio de la Escala de Estilos de Aprendizaje en Situaciones de Uso de Tecnologias; los datos sobre desempeño académico, a su vez, fueron obtenidos con base en evaluaciones realizadas en una disciplina de graduación en administración, utilizando la metodología activa de aprendizaje basada en problemas (PBL). Los resultados indicaron que el desempeño académico de los estudiantes de administración no dependia de sus estilos de aprendizaje, posiblemente en función de la diversidad de actividades realizadas en el marco de la metodología activa adoptada. Palabras clave: estilo de aprendizaje, rendimiento académico, tecnología de la información, universitarios, administración
Artigo está licenciado sob forma de uma licença Creative Commons Atribuição 4.0 Internacional. 
Os estilos de aprendizagem representam, em linhas gerais, o modo preferencial de aprender dos individuos. Tal conceito tem sido amplamente explorado na literatura científica (Dunn, Beaudry, \& Klavas, 2002; Kolb \& Kolb, 2005; Oliveira, Santos, \& Scacchetti, 2017). Compreendê-los pode, a priori, trazer vários benefícios para a educação, subsidiando os principais atores do processo de ensino e aprendizagem, ou seja, os estudantes, os professores e os gestores educacionais (Roza, 2017: Silva, Leal, Pereira, \& Oliveira Neto, 2015), em suas atividades escolares e acadêmicas.

Um dos grandes interesses associados aos estilos de aprendizagem é sua possivel relação com desempenho acadêmico. Contudo, Li e Tsai (2016) destacam que as conclusões das pesquisas sobre a relação entre essas variáveis são heterogêneas. Para ilustrar esta situação, a partir de uma revisão da literatura, os autores mencionam alguns estudos realizados sobre a temática, cujos principais resultados encontramse descritos na Tabela 1.

Conforme exemplificado na Tabela 1, os estudos sobre a relação entre estilos de aprendizagem e desempenho acadêmico utilizam diferentes instrumentos de medida. Nesses estudos, em particular, os instrumentos empregados foram o Learning Style Inventory, baseado no modelo aprendizagem experiencial (Kolb, 1984), o Productivity Environmental Preference Survey, derivado dos estudos de Dunn e Dunn (1978), o Learning Style Indicator (Wintergerst, DeCapua,

TABELA 1 - Estudos sobre a relação entre estilos de aprendizagem e desempenho acadêmico

\begin{tabular}{|c|c|c|c|}
\hline Estudo & Instrumento(s) & $\begin{array}{l}\text { Relação entre } \\
\text { estilos e } \\
\text { desempenho }\end{array}$ & $\begin{array}{l}\text { Contexto de uso } \\
\text { de TICs }\end{array}$ \\
\hline $\begin{array}{l}\text { Sucesso na educação à distância: } \\
\text { os estilos de aprendizagem e os } \\
\text { vários formatos são importantes? } \\
\text { (Battalio, 2009) }\end{array}$ & $\begin{array}{l}\text { Index of Learning Styles } \\
\text { (ILS) }\end{array}$ & Significativa. & Sim \\
\hline $\begin{array}{l}\text { Um estudo das relações entre } \\
\text { estilos de aprendizagem, tipos } \\
\text { de participação e desempenho } \\
\text { na aprendizagem de linguagens } \\
\text { de programação suportadas por } \\
\text { fóruns on-line (Shaw, 2012) }\end{array}$ & $\begin{array}{l}\text { Learning Style Inventory } \\
\text { (LSI) }\end{array}$ & Significativa. & Sim \\
\hline $\begin{array}{l}\text { A relação entre os estilos de } \\
\text { aprendizagem de estudantes lati- } \\
\text { nos e seu desempenho acadêmico } \\
\text { (Torres, 2014) }\end{array}$ & $\begin{array}{l}\text { Learning Style Inventory } \\
\text { (LSI) e Productivity En- } \\
\text { vironmental Preference } \\
\quad \text { Survey (PEPS) }\end{array}$ & Não significativa. & Não \\
\hline $\begin{array}{l}\text { Analisando o efeito de estilos de } \\
\text { aprendizagem e hábitos de estudo } \\
\text { de aprendizes à distância sobre } \\
\text { o desempenho de aprendizagem } \\
\text { (Cakiroglu, 2014) }\end{array}$ & $\begin{array}{l}\text { Learning Style Inventory } \\
\text { (LSI) }\end{array}$ & Significativa. & Sim \\
\hline $\begin{array}{l}\text { Estilos de aprendizagem e desem- } \\
\text { penho de estudantes em econo- } \\
\text { mia introdutória (Brunton, 2015) }\end{array}$ & $\begin{array}{l}\text { Learning Style Inventory } \\
\text { (LSI) }\end{array}$ & Não significativa. & Não \\
\hline $\begin{array}{l}\text { Um estudo sobre estilos de apren- } \\
\text { dizagem preferidos de formandos } \\
\text { de professores turcos de EFL (Inal, }\end{array}$ & Learning Style Indicator & Não significativa. & Não \\
\hline
\end{tabular}


\& Itzen, 2001), e o Index of Learning Styles, cujas bases estão no modelo de Felder e Silverman (1988).

Os instrumentos mencionados avaliam estilos de aprendizagem distintos, resultantes de diferentes classificações. A título de exemplo, o Learning Style Inventory considera quatro estilos de aprendizagem básicos, o acomodador, o assimilador, o convergente e o divergente (Kolb \& Kolb, 2005), enquanto o Index of Learning Styles (Soloman \& Felder, 2001) leva em consideração os estilos sensorial/intuitivo, visual/verbal, ativo/ reflexivo e sequencial/global, pertencentes às dimensões de percepção, entrada, processamento e compreensão, respectivamente.

É possivel observar que alguns dos referidos estudos indicam haver uma relação significativa entre estilos de aprendizagem e desempenho acadêmico, mas outros não. Em alguns deles, nota-se também que o contexto de uso das Tecnologias da Informação e Comunicação (TICs) se fez presente. Tais tecnologias referem-se ao conjunto de recursos de hardware e software, como computadores, smartphones, programas de modo geral e a Internet (Roza \& Wechsler, 2017). empregados no tratamento, na organização e na disseminação de informação (Takahashi, 2000).

Cabe ressaltar que as investigações sobre estilos de aprendizagem no contexto de uso das TICs são relativamente recentes. Ademais, os instrumentos de avaliação dos estilos de aprendizagem nestes contextos são escassos. Neste sentido, é possivel destacar, como instrumento, a Escala de Estilos de Aprendizagem em Situações de Uso de Tecnologias (Roza, Nakano, Silva, \& Wechsler, 2018; Roza, Nakano, Wechsler, \& Primi, 2018; Roza, Wechsler, \& Nakano, 2018). Tal escala é composta por quatro estilos de aprendizagem: o teórico, o pragmático, o de interação com o meio tecnológico e o estilo de interação através do meio tecnológico.

O estilo teórico da Escala de Estilos de Aprendizagem em Situações de Uso de Tecnologias refere-se à preferência de aprender por meio de modelos, teorias e conceitos e de reflexão, usando as TICs em atividades analíticas e abstratas. O estilo pragmático está associado a preferências por atividades práticas, empregando as tecnologias em situações reais e em problemas concretos. No estilo de interação com o meio, a preferência é pelo emprego das TICs para explorar coisas novas, de modo individual. No estilo de interação através do meio, a preferência de aprendizagem reside no uso das TICs para comunicação pessoal e em trabalhos colaborativos, com estabelecimento de relações sociais (Roza, 2017).

Além disso, as investigações científicas sobre a relação entre estilos de aprendizagem e desempenho acadêmico envolvem participantes com formações diversas e com diferentes niveis de escolaridade. Especificamente na área de ensino de administração, na qual se concentra o interesse do presente estudo, são encontrados estudos com participantes de nivel técnico (Walter, Fortes, Stettiner, \& Ramos, 2017), de graduação (Cordeiro \& Silva, 2012; Nogueira, Espejo, Reis, \& Voese, 2012) e de pós-graduação (Dias, Sauaia, \& Yoshizaki, 2013: Silva et al., 2015).

No âmbito de um curso de graduação em administração, por exemplo, Nogueira et al. (2012) investigaram se os desempenhos dos estudantes nas disciplinas de contabilidade geral e contabilidade gerencial, na modalidade de educação a distância (EaD), eram diferentes em função dos seus estilos de aprendizagem. Participaram do estudo 109 estudantes de administração de uma instituição de ensino superior pública federal brasileira. O instrumento utilizado para avaliação dos estilos de aprendizagem foi o Learning Style Inventory (Kolb \& Kolb, 2005). Os autores destacam que não houve diferença de desempenho associada ao estilo de aprendizagem dos estudantes.

A investigação de Dias et al. (2013), por sua vez, abordou os estilos de aprendizagem e o aprendizado no contexto de jogos de empresa. Buscou descrever e analisar, segundo os estilos, as diferenças de aprendizagem entre os estudantes de um curso de Planejamento e Controle da Produção (PCP). No total, foram considerados 356 estudantes de pós-graduação - Master in Business Administration (MBA) e especialização. Os estilos de aprendizagem foram avaliados a partir 
do Index of Learning Styles. O estudo revelou que o jogo de empresa, adotado na pesquisa, dinamizou as vivências, tendo sido detectado maior aprendizado por parte dos estudantes com estilo reflexivo ou visual, tomando como base o modelo de Felder e Silverman (1988).

Os resultados dos estudos sobre a relação entre as variáveis estilos de aprendizagem e desempenho acadêmico apontam para resultados heterogêneos. Enquanto algumas pesquisas destacam diferenças significativas de desempenho acadêmico em relação aos estilos de aprendizagem (Battalio, 2009; Cakiroglu, 2014; Dias et al., 2013: Shaw, 2012), outras indicam não haver diferenças estatisticamente significativas (Brunton, 2015; Inal et al., 2015; Nogueira et al., 2012; Torres, 2014). São muitas as razões para tais resultados, em especial as relacionadas ao fato de os estudos terem sido desenvolvidos em contextos e com instrumentos distintos, dificultando a comparação entre eles.

Diante deste cenário, Li e Tsai (2016) observam que o uso de diferentes instrumentos em contextos distintos parece produzir amplas discrepâncias nas descobertas sobre a relação entre estilos de aprendizagem e desempenho acadêmico, sugerindo a investigação dessa relação em estudos futuros. Além disso, considerando a evolução histórica das pesquisas sobre estilos de aprendizagem, nota-se um interesse crescente por situações que envolvem o uso de tecnologias. Nesse sentido, o presente estudo busca contribuir com as discussões sobre a temática, enfatizando o contexto de uso das TICs por estudantes de administração. Assim, em um primeiro momento, analisa os estilos de aprendizagem e o desempenho acadêmico dos estudantes separadamente. Posteriormente, como objetivo principal, investiga a relação entre os estilos de aprendizagem em situações de uso de tecnologias e o desempenho acadêmico de estudantes universitários de administração.

\section{Método}

\section{Participantes}

Os participantes foram 286 estudantes universitários de administração, de uma universidade privada do interior do estado de São Paulo, que cursaram uma disciplina de negócios em ambientes virtuais. Os estudantes possuíam idades entre 18 e 43 anos ( $M=21,97$; $D P=3,10)$, sendo 53,15\% ( $n=152)$ do gênero feminino. As linhas de formação dos estudantes foram administração "pura" ( $n=127)$. comércio exterior ( $n=120$ ) e logística e serviços $(n=39$ ).

\section{Instrumentos}

Escala de Estilos de Aprendizagem em Situações de Uso de Tecnologias - A escala em questão, desenvolvida por Roza (2017), é um instrumento composto por 40 itens. Em estudos anteriores, atingiu resultados satisfatórios em termos de evidências de validade baseadas no conteúdo (Roza, Wechsler et al., 2018) e foi informatizada (Roza, Nakano, Silva et al., 2018). Em estudo posterior foi estabelecida a evidência de validade baseada na análise da estrutura interna dos itens (Roza, Nakano, Wechsler et al., 2018). Para cada item da escala, o respondente deve indicar "Sim" ou "Não". Os estilos de aprendizagem contemplados pela escala são: teórico, pragmático, de interação com o meio e de interação através do meio.

Planilha de Avaliação de Desempenho Trata-se de uma planilha eletrônica para registro e cálculo de dados sobre desempenho acadêmico de estudantes de administração, na disciplina de negócios em ambientes virtuais, baseada na metodologia Problem Based Learning - PBL (Cachinho, 2012; Escrivão Filho \& Ribeiro, 2008; Toledo Júnior et al., 2008). Os dados sobre desempenho acadêmico contemplados na planilha são relativos às notas das atividades PBL e da prova final, realizadas na disciplina de negócios em ambientes virtuais.

\section{Procedimento}

A coleta de dados foi iniciada após a aprovação do Comitê de Ética da Pontifícia Universidade Católica de Campinas (PUC-Campinas), conforme parecer consubstanciado (CAAE: 56450716.3.0000.5481). Inicialmente, os estudantes receberam explicações sobre o estudo e foram convidados a participar da pesquisa. Aqueles que aceitaram, manifestaram-se de acordo com 
o Termo de Consentimento Livre e Esclarecido (TCLE) e, na sequência, responderam os itens da Escala de Estilos de Aprendizagem em Situações de Uso de Tecnologias, em meio eletrônico, de forma individual, durante o periodo de aula da disciplina de negócios em ambientes virtuais.

A coleta de dados sobre desempenho acadêmico, também, foi realizada ao longo das aulas da disciplina de negócios em ambientes virtuais, abrangendo notas de atividades PBL e prova final. As atividades avaliativas da metodologia PBL contemplaram a elaboração de relatórios parciais, pesquisas, apresentações, debates e entregas de relatórios finais. Elas foram realizadas não apenas em sala de aula, mas também em bibliotecas universitárias, laboratórios de informática e espaços de vivência gerencial.

Tendo em vista a posterior interpretação dos resultados obtidos, é importante esclarecer como ocorreram as atividades realizadas na disciplina, no que concerne àquelas da metodologia PBL. No decorrer da disciplina, os estudantes de administração dividiram-se em grupos. Tipicamente, cada grupo partiu de uma situaçãoproblema ou de uma proposta de estudo de caso. Os grupos atribuíram papéis a seus integrantes líder, redator, porta-voz e membros - e realizaram um relatório parcial, identificando os pontos-chave da situação-problema ou do estudo de caso, as estratégias de pesquisa e as responsabilidades de cada integrante. Posteriormente, realizaram as pesquisas propriamente ditas, utilizando-se do espaço e da estrutura das salas de aula, biblioteca universitária e laboratórios de informática. Por fim, apresentaram um relatório final e realizaram apresentações e debates entre si. Todas essas atividades foram acompanhadas por pelo menos um docente, que ao final do ciclo realizou uma aula expositiva de fechamento.

\section{Análise de dados}

Os dados obtidos por meio da Escala de Estilos de Aprendizagem em Situações de Uso de Tecnologias e da Planilha de Avaliação de Desempenho foram cruzados. Assim, foi obtido um banco de dados único, contendo informações sobre estilos de aprendizagem e desempenho acadêmico de estudantes universitários de administração de diferentes linhas de formação.

Para a análise dos dados sobre estilos de aprendizagem, foi realizada Estatística Descritiva, seguida de Análise Multivariada da Variância (Manova), sendo essa empregada na verificação da relação da variável linha de formação com os estilos de aprendizagem. $O$ desempenho acadêmico foi investigado por meio de Estatística Descritiva, Teste t de Student e Análise de Correlação de Pearson, contemplando as notas dos estudantes em PBL e na prova final. Por fim, para investigação da relação entre os estilos de aprendizagem e o desempenho acadêmico dos estudantes, foi realizada Análise de Correlação de Pearson. No caso das análises de Correlação de Pearson, foram adotados os critérios indicados por Dancey e Reidy (2013), que consideram valores de " $r$ " abaixo de 0,10 como nulos, de 0,10 e 0,39 como fracos, de 0,40 e 0,69 como moderados, de 0.70 a 0,90 como fortes e valores acima de 0,90 como correlações perfeitas.

\section{Resultados}

Tendo em vista o objetivo do presente estudo, as análises concentraram-se nos estilos de aprendizagem em situações de uso de tecnologias e no desempenho acadêmico dos estudantes universitários de administração. A Tabela 2 apresenta as pontuações brutas dos estudantes, por linha de formação, nos estilos de aprendizagem em situações das TICs.

Conforme mostrado na Tabela 2, os estudantes de administração com linha de formação em logística e serviços apresentaram as maiores pontuações médias em todos os estilos de aprendizagem. As menores médias nos estilos teórico e pragmático foram dos estudantes de administração com linha de formação em comércio exterior. Nos dois estilos de interação, as menores pontuações médias foram dos estudantes de administração "pura".

Para verificar a relação da variável linha de formação com os estilos de aprendizagem. foi realizada a Manova. Os seus resultados encontram-se na Tabela 3. 
TABELA 2 - Pontuações nos estilos de aprendizagem

\begin{tabular}{|c|c|c|c|c|c|c|c|}
\hline \multicolumn{3}{|c|}{ Curso/Linha de formação } & Teórico & Pragmático & $\begin{array}{l}\text { Interação } \\
\text { com o meio }\end{array}$ & \multicolumn{2}{|c|}{$\begin{array}{l}\text { Interação } \\
\text { através do } \\
\text { meio }\end{array}$} \\
\hline \multicolumn{3}{|c|}{ Minimo } & 0 & 2,0 & 0 & \multicolumn{2}{|c|}{1,0} \\
\hline \multirow{3}{*}{ Administração } & \multicolumn{2}{|c|}{ Máximo } & 13,0 & 10,0 & 8,0 & \multicolumn{2}{|c|}{8.0} \\
\hline & \multicolumn{2}{|c|}{ Média } & 7,024 & 8.913 & 6,071 & \multicolumn{2}{|c|}{4,693} \\
\hline & \multicolumn{2}{|c|}{$\begin{array}{l}\text { Desvio pa- } \\
\text { drão }\end{array}$} & 2,9452 & 1,5534 & 1,6192 & \multicolumn{2}{|c|}{1,8323} \\
\hline \multirow{4}{*}{$\begin{array}{l}\text { Administração com } \\
\text { linha de formação em } \\
\text { Comércio Exterior }\end{array}$} & \multicolumn{2}{|c|}{ Mínimo } & 0 & 4,0 & 2,0 & \multicolumn{2}{|c|}{1,0} \\
\hline & \multicolumn{2}{|c|}{ Máximo } & 13,0 & 10,0 & 8,0 & \multicolumn{2}{|c|}{8,0} \\
\hline & \multicolumn{2}{|c|}{ Média } & 6,842 & 8,883 & 6,117 & \multicolumn{2}{|c|}{4,833} \\
\hline & \multicolumn{2}{|c|}{$\begin{array}{l}\text { Desvio pa- } \\
\text { drão }\end{array}$} & 2,9563 & 1,3422 & 1,4505 & \multicolumn{2}{|c|}{1,5629} \\
\hline \multirow{4}{*}{$\begin{array}{l}\text { Administração com } \\
\text { linha de formação em } \\
\text { Logística e Serviços }\end{array}$} & \multicolumn{2}{|c|}{ Minimo } & 4,0 & 6.0 & 3.0 & \multicolumn{2}{|c|}{2,0} \\
\hline & \multicolumn{2}{|c|}{ Máximo } & 12,0 & 10,0 & 8,0 & \multicolumn{2}{|c|}{8,0} \\
\hline & \multicolumn{2}{|c|}{ Média } & 8,077 & 9,205 & 6.564 & \multicolumn{2}{|c|}{5.179} \\
\hline & \multicolumn{2}{|c|}{$\begin{array}{l}\text { Desvio pa- } \\
\text { drão }\end{array}$} & 2,0311 & 1,1045 & 1,4472 & \multicolumn{2}{|c|}{1,4118} \\
\hline & \multicolumn{2}{|c|}{ Minimo } & 0 & 2,0 & 0 & \multicolumn{2}{|c|}{1,0} \\
\hline & \multicolumn{2}{|c|}{ Máximo } & 13,0 & 10,0 & 8,0 & & 0 \\
\hline Total & Méd & & 7,091 & 8,941 & 6.157 & & 18 \\
\hline & $\begin{array}{l}\text { Desv } \\
\text { drão }\end{array}$ & pa- & 2,8627 & 1,4117 & 1,5309 & & 718 \\
\hline TABELA 3 - Manova & - difere & ça de & tilos em & TABELA 4 - Desem & mpenho aca & dêmico & \\
\hline relação à linha de for & naçăo & & & Curso/Linha de & & וחת & $\Omega_{0}$ \\
\hline & Qua- & & & formação & & $P D L$ & PTOVA \\
\hline ESEIIO & $\begin{array}{l}\text { ardáo } \\
\text { Médio }\end{array}$ & $r$ & sig. & & $\begin{array}{l}\text { Mini- } \\
\text { mo }\end{array}$ & 5,2 & 1,0 \\
\hline Pragmático & $\begin{array}{l}22,973 \\
1,608\end{array}$ & $\begin{array}{l}2,839 \\
0,806\end{array}$ & $\begin{array}{l}0,060 \\
0,448\end{array}$ & Administração & $\begin{array}{l}\text { Máxi- } \\
\text { mo }\end{array}$ & 8.7 & 10,0 \\
\hline $\begin{array}{l}\text { Interação com o } \\
\text { meio }\end{array}$ & 3,800 & 1,629 & 0,198 & & $\begin{array}{l}\text { Média } \\
\text { DP }\end{array}$ & $\begin{array}{l}7,070 \\
0,8146\end{array}$ & $\begin{array}{l}6,680 \\
1,6185\end{array}$ \\
\hline $\begin{array}{l}\text { Interação através do } \\
\text { meio }\end{array}$ & 3.556 & 1,275 & 0,281 & & $\begin{array}{l}\text { Mini- } \\
\text { mo }\end{array}$ & 4,9 & 2,5 \\
\hline$F=$ formação; Sig. = varia & ão signif & ativa. & & $\begin{array}{l}\text { Administração com } \\
\text { linha de forma- } \\
\text { ção em Comércio }\end{array}$ & $\begin{array}{l}\text { Máxi- } \\
\text { mo }\end{array}$ & 9,2 & 10,0 \\
\hline & & & & Exterior & Média & 7.399 & 6,608 \\
\hline & & & & & $\mathrm{DP}$ & 0,8349 & 1,6831 \\
\hline
\end{tabular}




\begin{tabular}{llcc} 
& Míni- & 4,6 & 0 \\
mo & & \\
$\begin{array}{l}\text { Administração com } \\
\text { linha de formação } \\
\text { em Logística e }\end{array}$ & Máxi- & 8,6 & 9,0 \\
Serviços & mo & & \\
& Média & 6,864 & 6,218 \\
& DP & 1,1771 & 1,9290 \\
Total & Mínimo & 4,6 & 0 \\
& Máximo & 9,2 & 10,0 \\
& Média & 7,180 & 6,587 \\
& DP & 0,8992 & 1,6911 \\
\hline
\end{tabular}

$\mathrm{PBL}=$ Problem Based Learning; $\mathrm{DP}$ = Desvio padrão

Segundo a Tabela 3, não houve diferença significativa de estilos de aprendizagem em relação à linha de formação. Desse modo, as análises subsequentes associadas aos estilos de aprendizagem dos estudantes de administração consideraram as pontuações totais, abrangendo todas as linhas de formação.

Após as análises relativas aos estilos de aprendizagem, foram analisados os dados sobre desempenho acadêmico. A Tabela 4 mostra o desempenho acadêmico dos estudantes de administração na disciplina de negócios em ambientes virtuais. $O$ desempenho acadêmico foi mensurado por meio de notas de 0 a 10, atribuidas às atividades PBL e à prova final realizadas na disciplina.

De acordo com a Tabela 4, a maior média em PBL foi dos estudantes de administração com linha de formação em comércio exterior. Na prova final, a maior média foi dos estudantes de administração "pura". Os estudantes de administração com linha de formação em logistica e serviços tiveram as médias mais baixas, tanto em $\mathrm{PBL}$ como na prova final.

Por linha de formação, a diferença absoluta entre a maior e a menor média em PBL foi baixa, assim como entre as médias na prova final. Em ambos os casos a diferença foi de aproximadamente meio ponto. Observa-se também que a variação das notas na prova final foi a máxima possivel. abrangendo todo o intervalo de 0 a 10

No total, a média das notas em PBL foi maior que a média de notas obtidas pelos estudantes na prova final. Para melhor compreender a relação entre as notas em PBL e na prova final, foram realizados o Teste $t$ de Student e a Análise de Correlação de Pearson. $O$ teste $t$ mostrou que a diferença entre as notas foi significativa $(t=5.597$ : $p<0,001)$. Os resultados da análise de correlação indicaram a existência de uma correlação significativa e de magnitude fraca entre as notas $(r=0,151 ; p=0,010)$, segundo os critérios indicados por Dancey e Reidy (2013).

Também foram realizados testes de correlação de Pearson para verificação da relação entre os estilos de aprendizagem e as notas dos estudantes de administração. A Tabela 5 apresenta os valores obtidos com os testes de correlação.

Conforme pode ser visto na Tabela 5, houve apenas uma correlação significativa entre o estilo pragmático e o desempenho dos estudantes nas atividades PBL. Contudo, observa-se que esta correlação foi fraca (Dancey \& Reidy, 2013).

TABELA 5 - Relação entre estilos de aprendizagem e desempenho acadêmico

\begin{tabular}{|c|c|c|c|c|c|}
\hline Avaliação & & Teórico & Pragmático & $\begin{array}{l}\text { Interação } \\
\text { com o meio }\end{array}$ & $\begin{array}{l}\text { Interação } \\
\text { através do } \\
\text { meio }\end{array}$ \\
\hline \multirow{2}{*}{ PBL } & Correlação de Pearson & 0,045 & $0,117^{*}$ & 0,051 & 0,015 \\
\hline & Sig. (2 extremidades) & 0,449 & 0,047 & 0,394 & 0,802 \\
\hline \multirow{2}{*}{ Prova } & Correlação de Pearson & $-0,048$ & $-0,066$ & $-0,016$ & 0,020 \\
\hline & Sig. (2 extremidades) & 0,422 & 0,264 & 0,782 & 0,742 \\
\hline
\end{tabular}

$\mathrm{PBL}=$ Problem Based Learning; Sig. = variação significativa.

* A correlação é significativa no nivel 0,05 (2 extremidades). 


\section{Discussão}

Ao revisar a literatura cientifica, foi possivel verificar que as pesquisas sobre a relação entre estilos de aprendizagem e desempenho acadêmico apresentam resultados heterogêneos (Li \& Tsai, 2016). Alguns estudos indicam uma relação significativa entre essas variáveis, enquanto outros não. Dentre as causas dessa heterogeneidade, possivelmente estão aspectos como diferenças de contexto, instrumentos e caracteristicas acadêmicas dos participantes envolvidos nos estudos, incluindo as suas áreas de formação e os seus niveis de escolaridade.

Por isso, é importante ressaltar que o presente estudo foi realizado no contexto de uso das TICS, tendo a Escala de Estilos de Aprendizagem em Situações de Uso de Tecnologias (Roza, Nakano, Wechsler et al., 2018; Roza, Wechsler et al., 2018) como instrumento aplicado a estudantes de graduação em administração, pertencentes a diferentes linhas de formação. Em comum, todos os estudantes cursaram uma disciplina de negócios em ambientes virtuais, cujas avaliações foram consideradas para mensuração de desempenho acadêmico.

A disciplina em questão foi baseada na metodologia PBL (Cachinho, 2012; Escrivão Filho \& Ribeiro, 2008; Toledo Júnior et al., 2008). Tanto a disciplina como a metodologia PBL tiveram um papel muito relevante na condução deste estudo. Elas foram escolhidas por permitirem um uso amplo e espontâneo de tecnologias por parte dos estudantes, conforme constatado por Roza e Wechsler (2017), fornecendo um ambiente rico de investigação.

Nesse sentido, cabe destacar que a metodologia PBL é considerada ativa, centrada no estudante (Cachinho, 2012). Ela parte do pressuposto de que a aprendizagem não é mera recepção de informação, mas sim um processo de construção de significados (Escrivão Filho \& Ribeiro, 2008). Assim, a disciplina baseada em PBL, considerada neste estudo, permitiu que os estudantes fossem protagonistas de seus processos de aprendizagem, inclusive nas situações de uso das TICs.
No que se refere aos estilos de aprendizagem em situações de uso de tecnologias, os estudantes de administração não apresentaram diferenças significativas de estilos em relação a suas linhas de formação. Esse resultado é coerente, à medida que as diferentes linhas de formação pertencem a um mesmo curso, com uma base curricular comum.

Quanto ao desempenho acadêmico, em média, as notas em PBL foram ligeiramente mais alta que as notas na prova final. Essa diferença foi estatisticamente significativa, o que permite afirmar que os estudantes tiveram um desempenho melhor nas atividades PBL. É interessante notar que as notas em PBL e as notas na prova final estão correlacionadas. Essa correlação pode ser explicada por meio da compreensão das atividades PBL como um processo, que abrange pesquisas, elaboração de relatórios, apresentações, debates e aulas expositivas e que possui os seus próprios critérios de avaliação, mas cujo resultado também é aferido, ao final, por meio de uma prova.

Contudo, a correlação entre as notas em $\mathrm{PBL}$ e as notas na prova final foi fraca. Algumas possiveis razões são: ao dividirem os papéis e responsabilidades entre os integrantes do grupo nas atividades PBL, os estudantes assimilaram parte do conteúdo esperado, não sendo essa lacuna totalmente superada durante as apresentações, debates ou aula de fechamento; a avaliação de desempenho individual nas atividades PBL foi parcialmente distorcida pelo desempenho do grupo; a prova final, como um instrumento de medição pontual, avaliou parte da multiplicidade de aspectos considerados na avaliação das atividades PBL.

Ao se investigar a relação entre estilos de aprendizagem e desempenho acadêmico, o estilo pragmático e as notas em PBL mostraram-se correlacionados, mesmo que de forma fraca. $O$ estilo pragmático refere-se às preferências de aprendizagem envolvendo aplicações práticas de ideais, um direcionamento para situações concretas (Roza, Nakano, Wechsler et al., 2018). Logo, tais preferências vão ao encontro das bases da metodologia PBL, que se apoiam 
em situações-problema e estudos de casos, conforme mencionado.

Ainda no que se refere à relação entre os estilos de aprendizagem e o desempenho acadêmico, é possivel apontar que as notas em PBL e na prova final não dependeram dos estilos dos estudantes de administração. Uma possivel explicação pode ser a diversidade de atividades PBL, que supostamente conseguiu atender às preferências individuais dos vários estilos de aprendizagem em situações de uso de tecnologia dos estudantes de administração.

Se por um lado as atividades diversificadas da disciplina considerada fornecem um ambiente favorável a diferentes estilos de aprendizagem, por outro, consistem uma limitação do presente estudo, à medida que não permitem a avalição dos estilos em um cenário delimitado. Nesse sentido, estudos com disciplinas predominantemente teóricas ou práticas, ou que adotem um único tipo de interação apoiada pelas TICs, poderiam revelar uma relação de dependência do desempenho acadêmico em função dos estilos de aprendizagem. Assim, sugere-se este tipo de abordagem, que privilegia teoria, prática ou uma forma específica de interação, em estudos futuros, tendo em vista o aprofundamento da compreensão sobre as relações entre os estilos de aprendizagem em situações de uso das tecnologias e desempenho acadêmico.

\section{Referências}

Battalio, J. (2009). Success in distance education: Do learning styles and multiple formats matter? American Journal of Distance Education, 23(2), 71-87. https://doi.org/10.1080/08923640902854405

Brunton, B. (2015). Learning styles and student performance in introductory economics. Journal of Education for Business, 90(2), 89-95. https://doi.org/1 $\underline{0.1080 / 08832323.2014 .980716}$

Cachinho, H. (2012). Criando experiências de aprendizagem significativas: do potencial da Aprendizagem Baseada em Problemas. El Hombre y la Máquina, 40, 58-67. Recuperado de https://WwW. redalyc.org/html/478/47826850007/
Cakiroglu, U. (2014). Analyzing the effect of learning styles and study habits of distance learners on learning performance: A case of an introductory programming course. International Review of Research in Open and Distance Learning, 15(4), 161185. https://doi.org/10.19173/irrodl.v15i4.1840

Cordeiro, R. A. \& Silva, A. B. (2012). Os estilos de aprendizagem influenciam o desempenho acadêmico dos estudantes de finanças? Revista de Administração da UFSM, 5(2), 243-261. https://doi. org/10.5902/198346594541

Dancey, C. P. \& Reidy, J. (2013). Estatística sem matemática para psicologia: usando o SPSS para Windows. Porto Alegre: Artmed.

Dias, G. P. P., Sauaia, A. C. A., \& Yoshizaki, H. T. Y. (2013). Estilos de aprendizagem Felder-Silverman e o aprendizado com jogos de empresa. Revista de Administração de Empresas, 53(5), 469-484. Recuperado de http://bibliotecadigital.fgv.br/ojs/ index.php/rae/article/view/30001/28852

Dunn, R. S. \& Dunn, K. J. (1978). Teaching students through their individual learning styles: a practical approach. Reston: Prentice Hall.

Dunn, R., Beaudry, J. S., \& Klavas, A. (2002). Survey of research on learning styles. California Journal of Science Education, 2(2), 75-98. Recuperado de http:// marric.us/files/CSTA_Learnjournal.pdf\#page=76

Escrivão Filho, E. \& de Ribeiro, L. R. C. (2008) Inovando no ensino de administração: uma experiência com a Aprendizagem Baseada em Problemas (PBL). Cadernos EBAPE.BR, 1-9. Recuperado de http://bibliotecadigital.fgv.br/ojs/ index.php/cadernosebape/article/view/5431/4165. https://doi.org/10.1590/S1679-39512008000500004

Felder, R. M. \& Silverman, L. K. (1988). Learning and teaching styles in engineering education. Engineering Education, 78(7), 674-681. Recuperado de http:// winbev.pbworks.com/f/LS-1988.pdf

Inal, S., Buyukyavuz, O., \& Tekin, M. (2015). A study on preferred learning styles of Turkish EFL teacher trainees. Australian Journal of Teacher Education, 40(3), 52-67. https://doi.org/10.14221/ajte.2014v40n3.4

Kolb, A. Y. \& Kolb, D. A. (2005). The Kolb learning style inventory - version 3.12005 technical specifications. Boston: Hay Resource Direct.

Kolb, D. A. (1984). Experiential learning: Experience as the source of learning and development. New Jersey: Prentice-Hall.

Li, C. L. K. \& Tsai, K. C. (2016). Personality, Learning Styles, Learning Motivation, and Academic Performance: A Study of Macau Business Undergraduates in a Microeconomics Course. European Journal of Business and Management, 8(21), 130-139. Recuperado de https://iiste.org/Journals/ index.php/EJBM/article/view/31680/32552 
Nogueira, D. R., Espejo, M. M. D. S. B., Reis, L. G., \& Voese, S. B. (2012). Estilos de aprendizagem e desempenho em educação a distância: um estudo empírico com alunos das disciplinas de contabilidade geral e gerencial. Revista de Educação e Pesquisa em Contabilidade (REPeC), 6(1), 54-72. https://doi. org/10.17524/repec.v6i1.181

Oliveira, K. L., Santos, A. A. A., \& Scacchetti, F. A. P. (2017). Evidências de validade para uma medida de estilos de aprendizagem. Revista Psicologia-Teoria e Prática, 19(3), 159-175. https://doi.org/10.5935/19806906/psicologia.v19n3p159-175

Roza, R. H. (2017). Estilos de aprendizagem e o uso das tecnologias da informação e comunicação. Tese de Doutorado, Pontificia Universidade Católica de Campinas.

Roza, R. H. \& Wechsler, S. M. (2017). O uso das tecnologias da informação e comunicação por estudantes de Administração. Competência - Revista da Educação Superior do Senac-RS, 10(2), 1-7. https:// doi.org/10.24936/2177-4986.v10n2.2017.523

Roza, R. H., Nakano, T. C., Silva, T. F., \& Wechsler, S. M. (2018). Tecnologia no Contexto Educacional: Teste Informatizado para Avaliação de Estilos de Aprendizagem. Psicologia da Educação (46), 11-20.

Roza, R. H., Nakano, T. C., Wechsler, S. M., \& Primi, R. (2018). Escala de Estilos de Aprendizagem em Situações de Uso de Tecnologias: estrutura interna. Avaliação Psicológica, 17(2), 223-232. https://doi. org/10.1568g/ap.2018.1702.14184.08

Roza, R. H., Wechsler, S. M., \& Nakano, T. C. (2018) Escala de estilos de aprendizagem em situações de uso de tecnologias: Busca por evidências de validade de conteúdo. Estudos Interdisciplinares em Psicologia, 9(1), 45-64. https://doi.org/10.5433/22366407.2018v9n1p45

Shaw, R. S. (2012). A study of the relationships among learning styles, participation types, and performance in programming language learning supported by online forums. Computers \& Education, 58(1), 111-120. https://doi.org/10.1016/i.compedu.2011.08.013

Silva, D. M., Leal, E. A, Pereira, J. M., \& Oliveira Neto, J. D. (2015). Estilos de aprendizagem e desempenho acadêmico na Educação a Distância: uma investigação em cursos de especialização. Revista Brasileira de Gestão de Negócios, 17(57), 1300-1316

Soloman, B. A. \& Felder, R. M. (2001). Index of learning styles questionnaire. Recuperado de http://WwW. engr.ncsu.edu/learningstyles/ilsweb.html

Takahashi, T. (Org.). (2000). Sociedade da informação no Brasil: livro verde. Brasília: Ministério da Ciência e Tecnologia

Toledo Júnior, A. C. C., Ibiapina, C. D. C., Lopes, S C. F., Rodrigues, A. C. P., \& Soares, S. M. S. (2008). Aprendizagem baseada em problemas: uma nova referência para a construção do currículo médico. Revista Médica de Minas Gerais, 18(2), 123-131. Recuperado de http://rmmg.org/artigo/detalhes/521
Torres, S. M. (2014). The relationship between Latino students' learning styles and their academic performance. Community College Journal of Research and Practice, 38(4), 357-369. https://doi.org/ $\underline{10.1080 / 10668926.2012 .761072}$

Walter, C. E., Fortes, P. J., Stettiner, C. F., \& Ramos, D. F. (2017). A influência dos estilos de aprendizagem no desempenho de grupos de trabalho. Journal of Learning Styles, 10(20), 156-181. Recuperado de http://learningstyles.uvu.edu/index.php/jls/article/ view/339/240

Wintergerst, A. C., DeCapua, A., \& Itzen, R. (2001). The construct validity of one learning styles instrument. System, 29, 385-403. Recuperado de https://WwW.sciencedirect.com/science/article/pii/ So346251X01000276?via\%3Dihub

\section{Endereço para correspondência:}

Rodrigo Hipólito Roza

Centro de Economia e Administração (CEA)

Rod. Dom Pedro I, Km 136 - Parque das Universidades 13086-900 - Campinas, SP, Brasil

\section{Rodrigo Hipólito Roza}

Pós-Doutorado em Psicologia pela Universidade São Francisco, em Campinas, SP, Brasil. Professor do Centro de Economia e Administração da Pontificia Universidade Católica de Campinas, em Campinas, SP, Brasil.

\section{Acácia Aparecida Angeli dos Santos}

Doutora em Psicologia pela Universidade de São Paulo, em São Paulo, SP, Brasil. Professora da PósGraduação Stricto Sensu em Psicologia da Universidade São Francisco, em Campinas, SP, Brasil. Bolsista de Produtividade em Pesquisa do CNPq. 